\title{
Effects of Female Genital Schistosomiasis in Reproductive Tract of Women Attending Kwale Hospital in Kwale County, Kenya
}

\author{
Jane M. N. Gitau ${ }^{1 *}, \mathrm{Ng}^{\prime}$ ethe Muhoho ${ }^{1}$ and Ephantus Kabiru ${ }^{2}$ \\ ${ }^{1}$ Department of Pathology, Kenyatta University, Kenya. \\ ${ }^{2}$ School of Public Health, Kenyatta University, Kenya.
}

\begin{abstract}
Authors' contributions
This work was carried out in collaboration between all authors. Author JMNG carried out data collection and analyses. Authors NM and EK designed and supervised the study. All authors read and
\end{abstract} approved the final manuscript.

Article Information

DOI: $10.9734 / \mathrm{IJTDH} / 2017 / 32787$

Editor(s):

(1) Janvier Gasana, Department of Environmental \& Occupational Health, Robert Stempel College of Public Health \& Social Work, Florida International University, USA.

Reviewers:

(1) Chester Kalinda, University of KwaZulu-Natal, South Africa.

(2) David Zadock Munisi, Nelson Mandela African Institution of Science and Technology, Tanzania.

(3) Anonymous, Oslo university Hospital, Oslo, Norway. (4) Awosolu, Oluwaseun, Federal University of Technology, Akure, Nigeria.

(5) Ali Mahmud Umar, Kano University of Science and Technology, Wudil, Kano State, Nigeria (6) Rodrigue Nana Dongang, Institute of Medical Research and Medicinal Plants Studies, Cameroon. Complete Peer review History: http://www.sciencedomain.org/review-history/19593

Original Research Article

Received $15^{\text {th }}$ March 2017

Accepted $1^{\text {st }}$ June 2017

Published 17 $7^{\text {th }}$ June 2017

\begin{abstract}
Background: Female genital Schistosomiasis (FGS) caused by Schistosoma haematobium is a significant public health problem among women living in endemic areas. An estimated 45 million women in sub-Saharan Africa are infected. Chronic infection is associated with bleeding disturbances, abdominal-pelvic pain, painful sexual intercourse, uterine fibroids, spontaneous abortion, cervical cancer and increased risk for transmission of HIV.

Aim: To determine the infection rate, distribution pattern and associated genital pathology in $S$. haematobium infection among females of reproductive age in endemic area in Kwale County, Kenya.

Study Design: Descriptive cross-sectional.

Study Area: Kwale level four hospital. Study subjects came from the hospital catchment area.

Methodology: Prevalence of $S$. haematobium was determined through urine filtration and
\end{abstract}


microscopy for $S$. haematobium eggs and by detection of both haematuria and proteinuria using strip reagent analysis. Intensity of $S$. haematobium infection was determined by scoring the number of eggs per $10 \mathrm{ml}$ of urine. Signs of genital pathology were determined through visual assessment of the genitalia by a gynaecologist. SPSS version 20.0 was used to analyze data.

Results: Overall prevalence of $S$. haematobium was $23 \%$. Intensity of infection was low with majority of infected participants (15.8\%) carrying light intensity infections ( $\leq 50 \mathrm{eggs} / 10 \mathrm{ml}$ of urine). Prevalence and intensity of infection was significantly high in women age group 18-23; $\left(X^{2}=5.778\right.$; $P=0.017)$. Signs of genital pathology were recorded in $46.7 \%$ of infected women. The results of this study indicate that female genital schistosomiasis is an important health problem in Kwale County and there is need for the Ministry of Health and the County government of Kwale to improve diagnosis and treatment of the disease as well as initiate strategies to control this problem.

Keywords: Prevalence; female genital schistosomiasis; distribution pattern; genital pathology; lesions.

\section{INTRODUCTION}

Schistosomiasis remains one of the most serious and prevalent diseases worldwide with an estimated 207million people infected [1]. About 160 million of infected cases are found in SubSahara Africa [2]. Schistosomiasis is a disease of the poor in the developing world where access to safe drinking water and sanitation is poor [1]. The disease is a major cause of morbidity and premature mortality in endemic areas of the world [3].

In Kenya, more than 6 million people are infected with urogenital or intestinal schistosomiasis caused by $S$. haematobium and $S$. mansoni respectively [4]. In the year 2003 a pilot control program was initiated in 86 schools in endemic areas where a total of forty thousand $(40,000)$ school age children were involved in a yearly treatment by trained school teachers for 4 consecutive years [5]. The success of this pilot program led to implementation of a National school-based de-worming program targeting more than 8 million school age children in all endemic areas. These control programs did not include adult population, especially the most vulnerable group such as women of reproductive age [6] as well as other adults at high risk such as farmers working in irrigation schemes and freshwater fishermen $[7,8]$.

An estimated 9-13 million women are afflicted with urogenital schistosomiasis in sub-Saharan Africa [9]. Up to $75 \%$ of women excreting $S$. haematobium eggs in the urine may have female genital schistosomiasis [10-13]; and up to $41 \%$ of women in endemic areas have schistosomiasis of the lower reproductive tract without eggs in urine [10]. Studies have shown that $S$. haematobium eggs may be distributed in all the pelvic organs with cervix being the most commonly affected, followed by the vagina, ovaries, fallopian tubes, vulva and the uterus [14]. Urogenital Schistosomiasis has been associated with bleeding disturbances, abdominal-pelvic pain, painful sexual intercourse, uterine fibroids, spontaneous abortion, cervical cancer and increased risk for transmission of HIV $[11,12,15]$.

Kwale County in Coastal Kenya has been endemic for $S$. haematobium since 1981 when the first baseline study on prevalence and intensity of infection was conducted and recorded as $68.2 \%$ and 50.0 eggs/hour respectively [16]. Despite the heavy burden of the disease on human population in endemic areas of Kenya, only limited control measures and programmes have been put in place. Most of them are school-based targeting primary school children, while others are sporadic projects and short term, targeting a certain community. In Kwale the risk of $S$. haematobium reinfection and late disease after school-based treatment is high. Diagnosis of infection is routinely based on presence of eggs in the urine [17]. It is possible that diagnosis and treatment of genital schistosomiasis is not adequately carried out leading to chronic illness among infected females. This research therefore focuses on the infection rate, distribution pattern and genital involvement of $S$. haematobium infection among females of reproductive age in Kwale County, Kenya with an aim to improve diagnosis and treatment of the disease and its effects on the genital tract.

\section{MATERIALS AND METHODS}

\subsection{Study Design}

This study adopted a descriptive cross-sectional design with study subjects being female patients 
visiting Kwale level four Hospital with gynaecological complaints.

\subsection{Study Area}

The site of this study was Kwale level four Hospital, located in Kwale Town, Kwale County, Kenya. Kwale County is in South Coast of Coast Province, Kenya. Drainage system is poor and consists of slow running streams. Stationary ponds and shallow open dams for animal watering are common. Such conditions provide favourable habitats for development and survival of freshwater snails associated with transmission of $S$. haematobium in the area.

\subsection{Study Population}

The study population comprised 196 women in their reproductive age (18-49 years) who visited Kwale Hospital with gynaecological problems. They were drawn from Kwale hospital catchment areas namely Tsimba/Vuga, Chirimani, Mtchang atamu, Mwachinga, Mwaluphamba and Ngonzini.

\subsection{Sampling Techniques}

The study employed a non-probability sampling techniques. Purposive sampling was used to select respondents who fit the criteria of the study population i.e. adult female patients of reproductive age who visited the hospital with gynaecological complaints.

\subsection{Data Collection Techniques}

\subsubsection{Prevalence and intensity of urogenital schistosomiasis}

Prevalence and intensity of urogenital schistosomiasis was accomplished by detection of $S$. haematobium eggs under microscopy and detection of both proteinuria and haematuria by urinalysis reagent strip. All participants with urine containing $S$. haematobium eggs and/or positive urinalysis test were treated with praziquantel 40 $\mathrm{mg} / \mathrm{kg}$ body weight [18].

Each participant was given a sterile $40 \mathrm{ml}$ universal bottle to provide terminal urine between 10.00 am and $1.00 \mathrm{pm}$. Each bottle was labeled to correspond to the study identification number issued to each participant. From each participant, $10 \mathrm{ml}$ urine specimen was filtered from the sample using Nuclepore urine filtration kit as described by Kahama et al. [18]. Using a blunt ended forceps the filter was transferred to a slide. A drop of physiological saline was added and then covered with a cover glass and examined under a microscope using X10 objectives. The number of $S$. haematobium eggs was counted and reported as the number of eggs per $10 \mathrm{ml}$ of urine, reflecting the intensity of $S$. haematobium infection. Intensity of infection was categorized as light if the eggs were $\geq 50$ per $10 \mathrm{ml}$ of urine, and heavy if eggs were $\leq 50$ per $10 \mathrm{ml}$ of urine [2].

Urinalysis test for presence of blood and proteins in urine was done using Uro-Labstix 3 (MilesSankyo Co. Ltd). All participants with negative urinalysis and parasitological tests were used as controls. A cellulose strip was dipped in a urine specimen contained in a well labeled sterile test tube. After a few seconds the strip was removed and observed for color changes. A change from light yellow to a range of blue colors indicated presence of haemoglobin while blue spots indicated intact erythrocytes. Any change of color to green indicated presence of proteins [17].

\subsubsection{Gynaecological examination for genital involvement}

The women were invited to undergo a gynaecological examination by a Gynaecologist. Examination of external genitalia, vagina and cervix was done (using disposable speculae). If pathological changes were observed in the cervix and the patient consented, cervical cancer screening tests were done using visual inspection methods. These were; Visual Inspection with 5\% Acetic Acid (VIA) and Visual Inspection with Lugol's lodine (VILI). All participants who tested positive with either VIA or VILI were referred appropriately to the next level hospital.

\subsection{Data Analysis}

The SPSS 20 version was used for data analysis. Descriptive statistics was done by running frequencies, means and crosstabulations. Chi-square test with $95 \%$ confidence intervals (Cls) was used to compare prevalence and intensity of $S$. haematobium infection between cases and controls while two sample ttest was used to compare lesions between the two study groups.

\subsection{Ethical Considerations}

The study protocol was reviewed and approved by Kenyatta University Ethics Review Committee 
(KU-ERC). Further clearance was sought from Ministry of Health Kwale County and from Kwale Hospital Medical Superintendent. Thereafter, informed written consent was obtained from each study subject.

\section{RESULTS}

\subsection{Distribution}

S. haematobium Infection among Participants

A total of 196 women were examined for $S$. haematobium infection with age ranges between 18 to 49 years. Most of the subjects $(50.5 \%)$ were housewives in age group 30-35 years and majority (17.8\%) came from TsimbaVuga area which is very close to Kwale town.

The overall prevalence of $S$. haematobium infection was $23 \%$. Infection was significantly high $\left(x^{2}=5.778 ;\right.$ df $\left.=4 ; P=0.017\right)$ among younger women aged 18-23 (35.3\%) compared to other age groups (Fig. 1). Most of the infected subjects were fishmongers (37.5\%) while women in formal employment had no infection (Fig. 2). Chirimani village recorded the highest infection rate $(36.4 \%)$ followed by Tsimba-Vuga while Mwachinga recorded the least (Fig. 3).

\subsection{Intensity of $S$. haematobium among the Study Participants}

Intensity of infection was low; women with light intensity infection were $15.8 \%$ while $7.1 \%$ had heavy intensity infection. Younger women aged 35 years and below had significantly $\left(x^{2}=4.077\right.$; df $=4 ; P=0.018)$ higher intensity infection compared to those aged 36 years and above (Fig. 4).

\subsection{Schistosoma haematobium Infection and Genital Pathology}

A total of $46.7 \%$ of women with S. haematobium infection had signs of genital pathology as follows; genital ulcers $17.8 \%$, contact bleeding $20 \%$, sandy patches $20 \%$ and tumor-like lesions $8.9 \%$ (Fig. 5). Two-Sample t-test showed that the mean number of sandy patches, tumorous lesions and bleeding sites was significantly higher in positive women $(\mathrm{t}=3.382 ; P=0.004, \mathrm{t}$ $=2.246 ; P=0.013$ and $\mathrm{t}=2.138 ; P=0.017$ ) respectively. Distribution of ulcers was not associated with $S$. haematobium infection (Fig. 5).

\section{DISCUSSION}

Findings of this study indicate that S. haematobium infection is still a health concern in Kwale County, Kenya. Infections are sustained by the high presence of Bulinus species of freshwater snails which are the vectors for S. haematobium [19]. Earlier studies reported a high prevalence of infection in the area; $33.4 \%$ [6], $18.2 \%$ [20], 43.2\% [21], 32.4\% [22] and $53.8 \%$, [23]. Infection rate in the current study was significantly higher in women of younger age group 18-23 and 24-29 years.

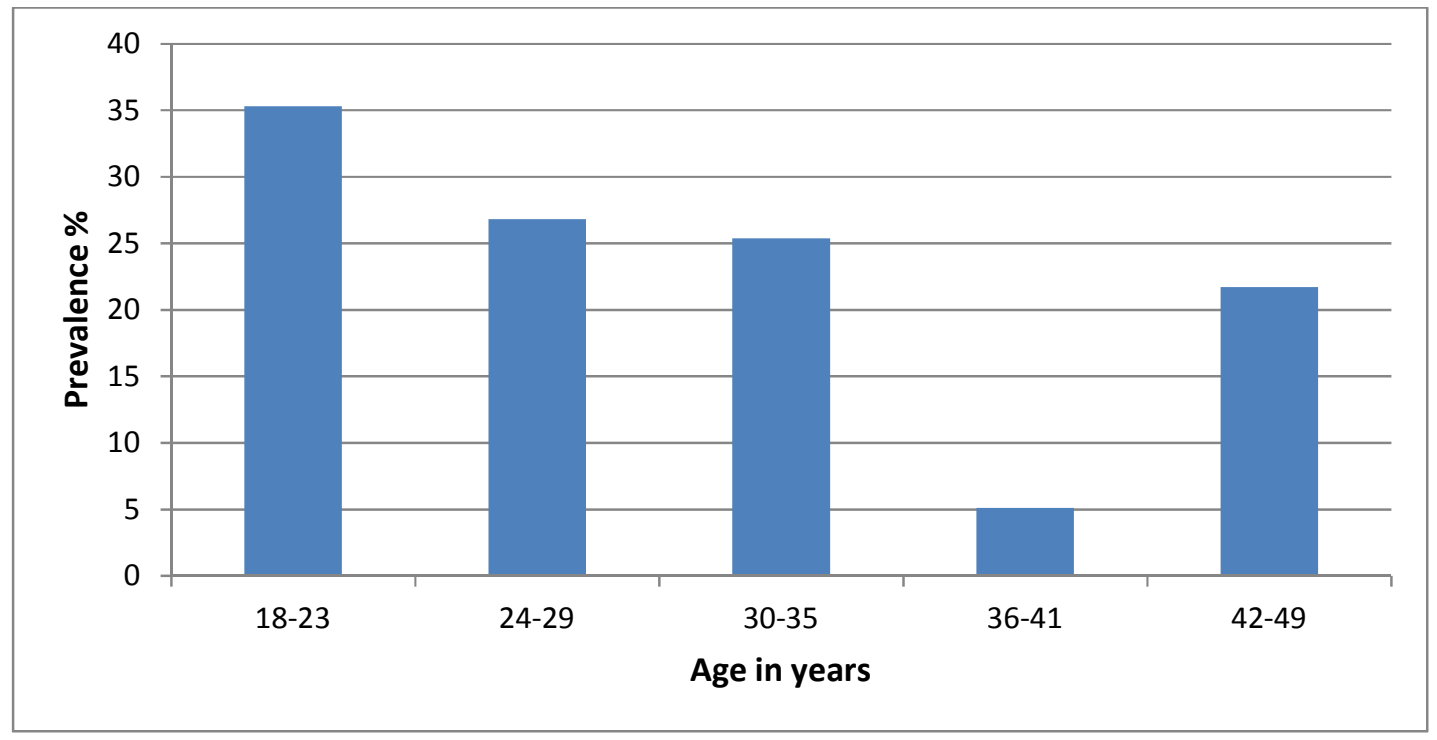

Fig. 1. Prevalence of S. haematobium infection by age 


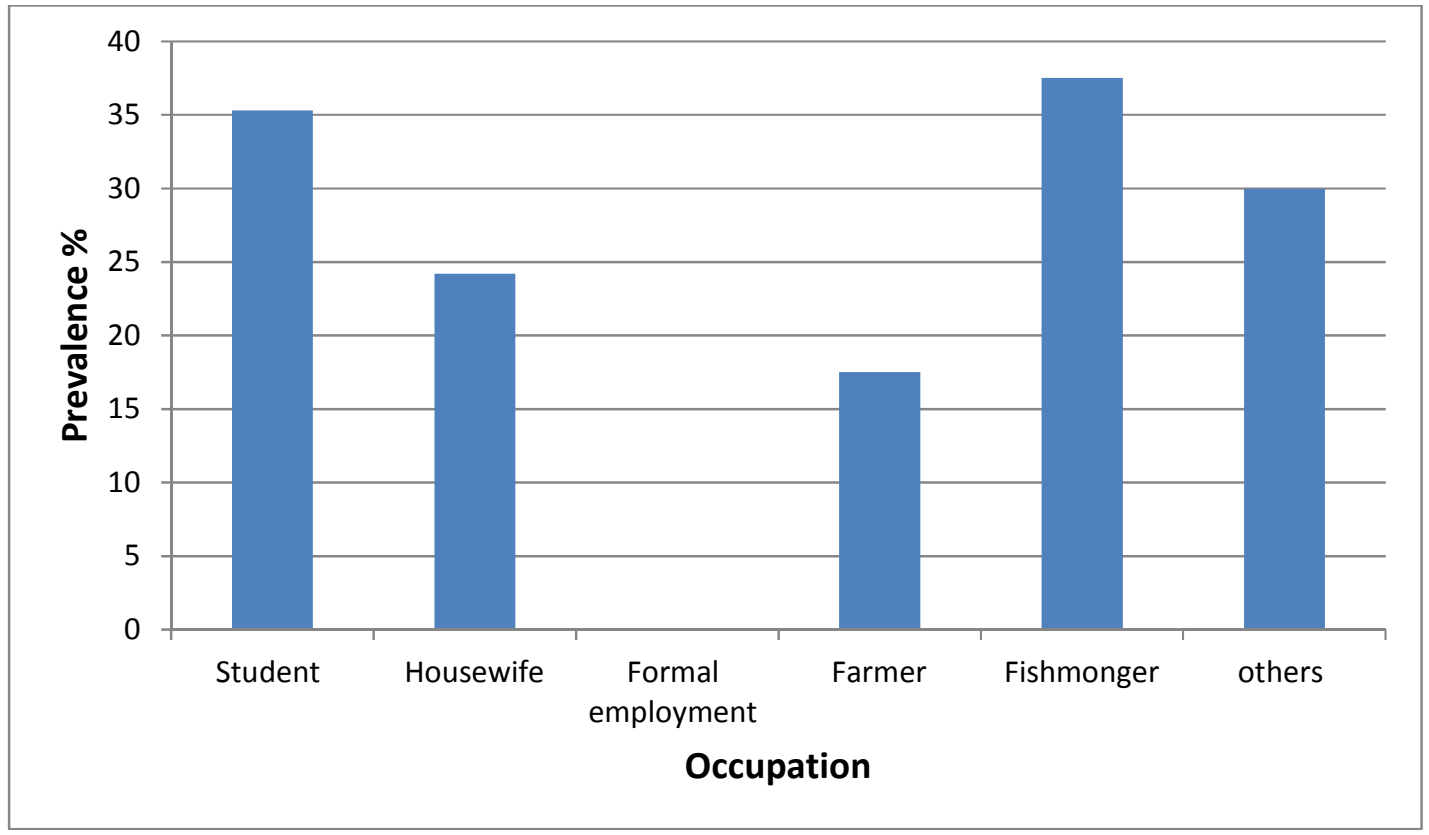

Fig. 2. Prevalence of $S$. haematobium infection by occupation

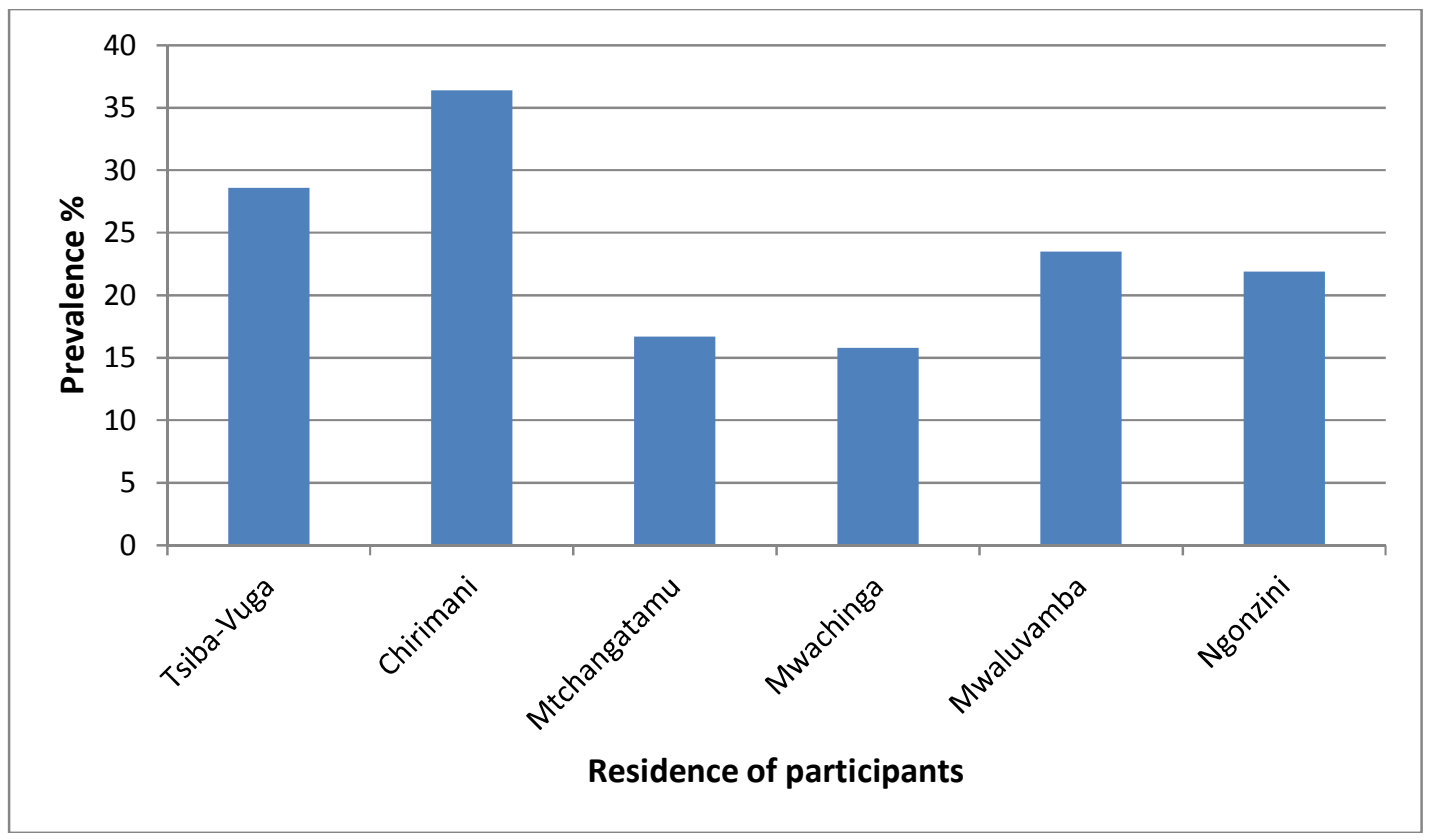

Fig. 3. Prevalence of $S$. haematobium infection by residence

Earlier studies associated such high infection rates to raised water contact activities in younger age groups [16,24]. Majority of infected women in the current study were fishmongers. It is expected that fishmongers and housewives have higher water contact level in course of their daily chores as compared to women in other occupations. According to a report by WHO, 2016 [7] adults considered to be at risk in endemic areas are people with occupations involving contact with infested water, such as fishermen, farmers, irrigation workers, and women whose domestic tasks bring them into contact with snail infested water $[7,8,24,25]$. 


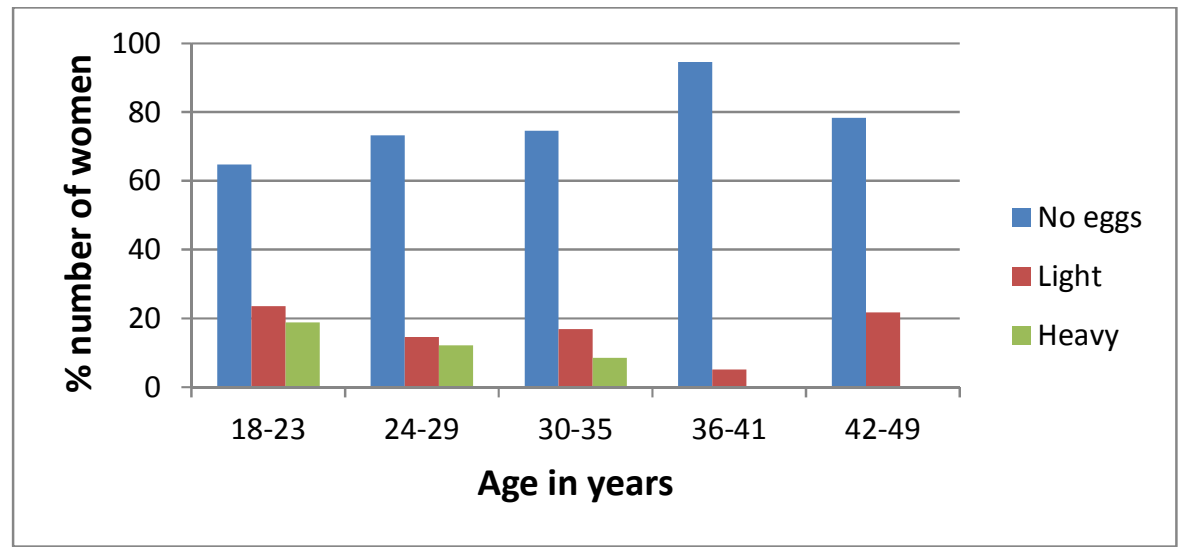

Fig. 4. Intensity of S. haematobium infection among the women

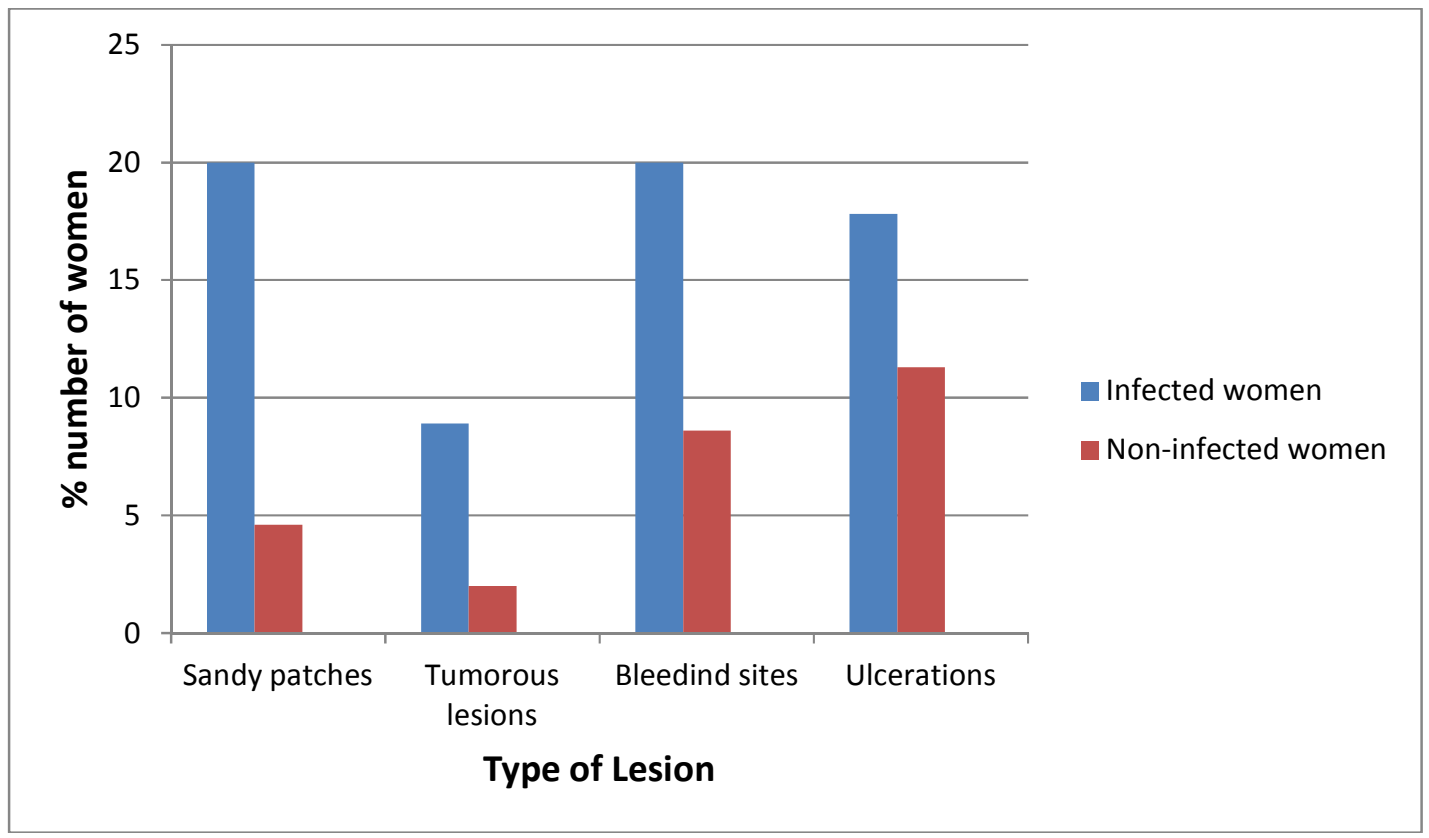

Fig. 5. Genital lesions and contact bleeding in infected and non-infected women

Prevalence and intensity of $S$. haematobium varied among villages with Chirimani and TshibaVuga area demonstrating higher prevalence than other areas $36.4 \%$ and $28.6 \%$ respectively. This could be attributed to the local streams that are perennial and slow running hence local people are continuously exposed to the infection all year round. Secondly there are no major control programmes in Chirimani, Tsimba and Vuga area. Considering the chronic nature of schistosomiasis, the women in these villages could have been suffering from the infection for a long time without seeking treatment. Another study in Kwale observed high infection with $S$. haematobium in some villages and attributed it to continuous contact with snail infested water and consistent lack of intervention such as mass de-worming in the area [6]. Lower infections were observed in Mtsangatamu and Mwachinga villages. These are in Kwale hinterland and had a long exposure to schistosomiasis control programmes [26,27]. Earlier projects provided piped water to the community in Mtsangatamu and a community dispensary in Mwachinga village where praziquantel (drug of choice for treatment of schistosomiasis) up to now has been included in the medicine kit $[26,27]$.

Intensity of infection was found to be high in younger women aged 35 years and below. 
Women in this age bracket who live in schistosomiasis - endemic regions could spend most of their reproductive life either pregnant or lactating, hence delayed administration of praziquantel coupled with low immunity to infections [6]. Delay in treatment of more than one year could lead to heavy infection and significant morbidity. Other research findings indicate that intensity of infection follows the same trend as prevalence whereby the younger ages of $16-35$ years consistently harbor heavier parasitic burdens, as measured by parasite egg load $[28,29]$.

Genital abnormalities were recorded in $46.7 \%$ of the infected women, spread on the cervix and the vaginal wall. This was a strong pointer to female genital schistosomiasis morbidity due to chronic infection. Poggensee et al. [13] observed that pathological lesions in the urogenital system in heavy $S$. haematobium infection were important hidden morbidity. Other clinical studies of women living in areas where $S$. haematobium was endemic yielded similar findings [30-32]. The current study identified tumorous lesions in $3.6 \%$ of women participants. These lesions were significantly more in the $S$. haematobium infected women. Recently, more additional cases of female genital malignancy in association with evidence of $S$. haematobium infection have been published [32].

\section{CONCLUSION}

Female genital schistosomiasis among women of reproductive age is high in Kwale and there is need to conduct proper community diagnosis and treatment of the disease.

\section{RECOMMENDATIONS}

The County government of Kwale in collaboration with the national government should put control programmes in place and enhance community sensitization on transmission and prevention of the disease.

\section{CONSENT}

As per international standard or university standard, patient's written consent has been collected and preserved by the authors.

\section{ETHICAL APPROVAL}

As per international standard or university standard, written approval of Ethics committee has been collected and preserved by the authors.

\section{ACKNOWLEDGEMENT}

The authors sincerely thank the women of Kwale for participating in this study and the Kwale hospital staff for their contribution and support.

\section{COMPETING INTERESTS}

Authors have declared that no competing interests exist.

\section{REFERENCES}

1. Awosolu OB. Epidemiology of urinary schistosomiasis and knowledge of health personnel in rural communities of SouthWestern Nigeria. J. of Parasit. and Vector Biol. 2016;8(10):99-106

2. WHO. Prevention and control of schistosomiasis and soil transmitted helminthiasis. Report of a WHO expert committee. Technical Report series 912; 2002.

3. King $\mathrm{CH}$, Dangerfield-Cha M. The unacknowledged impact of chronic schistosomiasis. Chronic Illness. 2008; 4(1):65-79.

4. Chitsulo L, Engels D, Montresor A, Savioli $\mathrm{L}$. The global status of schistosomiasis and its control. Acta Tropical. 2000;77:41-45.

5. Kihara JH, Muhoho N, Njomo D. Drug efficacy of praziquantel and albendazole in school children in Mwea Division, central province, Kenya. Acta Tropic. 2007; 102:165-172.

6. Kihara. Schistosoma haematobium infection in school children and women of reproductive age: The effect on anaemia, blood pathophysiological changes in selected parts of Kwale, Kilifi and birth weight outcomes in Tana River, County, Coast Province, Kenya; 2012.

7. WHO. Schistosomiasis. Fact sheet; 2016. Updated February 2016.

8. Bolaji OS, Adeyeba OA, Ojurongbe $\mathrm{O}$, Odewale G, Ukaga CN. Water contact activities and socio- cultural factors on urinary schistosomiasis in rural area of Osun State, Nigeria; 2014.

9. WHO, Schistosomiasis. Fact sheet no.115; 2010.

Available:http//www.who.int/mediacentre/fa ctsheets/fs 115/en/index.html

(Accessed on February 11, 2011) 
10. Kjetland EF, Ndhlovu PD, Mduluza T, Midzi N, Gwanzura L, Mason PR, Sandvik L, Friis H, Gundersen SG. Simple clinical manifestations of genital Schistosoma haematobium infection in rural Zimbabwean women. Amer. J. of Tropic. Medic. and Hygiene. 2005;72:311-9.

11. Leutscher P, Ravaoalimalala VE, Raharisolo C, Ramarokoto CE, Rasendramino M, Raobelison A, Vennervald $B$, Esterre $\mathrm{P}$, Feldmeier $\mathrm{H}$. Clinical findings in female genital schistosomiasis in Madagascar. Tropic. Med. \& Intern. Health. 1998;3:327-332. DOI: 10.1046/j.1365-3156.1998.00230

12. Renaud G, Devidas A, Develoux M, Lamothe F, Bianchi G. Prevalence of vaginal schistosomiasis caused by $S$. haematobium in an endemic village in Niger. Trans. Roy. Soc. Tropic. Med. Hygiene. 1989;38:797.

13. Poggensee G, Feldmeier H, Krantz I. Schistosomiasis of the Female Genital Tract; Public health aspect. Parasit. Today. 1999;15(9):378-381.

14. Kjetland EF, Kurewa EN, Ndhlovu PD, Midzi N, Gwanzura L, Mason PR, Gomo E, Sandvik L, Mduluza T, Friis H, Gundersen SG. Female genital schistosomiasis-a differential diagnosis to sexually transmitted disease: Genital itch and vaginal discharge as indicators of genital Schistosoma haematobium morbidity in a cross-sectional study in endemic rural Zimbabwe. Tropic. Med. and Intern. Health. 2008;13:1509-1517.

15. Kjetland EF, Leutcher PDC, Patricia D, Ndhlovu PD. A review of female genital schistosomiasis. Trends in Parasit. 2012; 28(2).

16. Shimada $\mathrm{M}$, Hirata $\mathrm{M}$, Ouma $\mathrm{JH}$, Wambanyi E, Thiong'o FW, Aoki Y. Epidemiological study of Schistosoma haematobium infection in the coastal area of Kenya. Japan J. of Tropic. Med. and Hygine. 1987;15(3):173-184.

17. Cheesbrough $M$. District laboratory practice in tropical countries part 2 . Cambridge Low price Edition. $2^{\text {nd }}$ Edition; 2007.

18. Kahama Al, Kremsner PG, Van dam GJ, Deelder AM. The dynamic of soluble egg antigen of Schistosoma haematobium in relation to egg counts, circulating anodic and cathodic antigen and pathology markers before and after chemotherapy.
Transact. of the Royal Soc. of Tropic. Med. and Hygiene. 1998;92:629-633.

19. Terer CC. Evaluation of health-related quality of life of children living with urinary schistosomiasis using PedsQL'M 4.0 SF15 TM and effects of the disease on iron levels in infected children in Kwale County, Kenya; 2012.

20. Njenga SM, Mwandawiro CS, Muniu E, Mwanje MT, Haji FM, Brokarie MJ. Adult population as potential reservoir of NTD infections. Parasites and Vectors. 2011; 4:175.

21. Mutuku FM, King $\mathrm{CH}$, Bustinduy $\mathrm{AL}$, Mungai PL, Muchiri EM, Kitron U. Impact of drought on the spatial pattern of transmission of Schistosoma haematobium in coastal Kenya. Amer. J. of Tropic. Med. and Hygine. 2011;85:1065-1070.

22. Clennon J, Mungai P, Muchiri E, King C, Kitron U. Spatial and temporal variations in local transmission of Schistosoma haematobium in Msambweni, Kenya. Amer. J. of Tropic. Med. and Hygine. 2006; 75:1034-1041.

23. Clennon JA, King $\mathrm{CH}$, Muchiri EM, Kariuki HC, Ouma JH, Mungai P, Kitron U. Spatial patterns of Urinary schistosomiasis infection in a highly endemic area of Coastal Kenya. Amer. J. of Tropic. Med. and Hygiene. 2004;70:443-448.

24. Njoku JC, Ajayi JA, Pitman SL, Dakul DA, Njoku OM. Urogenital schistosomiasis in females from some suburban communities of Jos, North Central Nigeria IOSR J. of Pharmacy and Biolog. Sci. (IOSR-JPBS) e2014;9:(3 Ver. III):69-79

ISSN: 2278-3008, ISSN: 2319-7676

25. Kabuyaya M, Chimbari MJ, Manyangadze $\mathrm{T}$, Mukaratirwa S. Schistosomiasis risk factors based on the infection status among school-going children in the Ndumo area, uMkhanyakude district, South Africa. Southern Afric. J. of Infectious Diseases. 2017;1-6.

26. Kaneko S, K'opiyo J, Kiche I, Wanyua S, Goto K, Tanaka J, Mwatasa C, Ndemwa M, Komazawa O, Karama M, Moji K, Shimada M. Health and demographic surveillance system in the western and coastal areas of Kenya: An infrastructure for epidemiologic studies in Africa; 2012.

27. Abe M, Muhoho ND, Sunahara T, Mojl K, Yamamoto T, Aoki Y. Effect of communal piped water supply on pattern of water use and transmission of schistosomiasis haematobia in an endemic area of Kenya. 
Tropic. Med. and Health. 2009;37(2):1929.

28. Jennifer AD, Charles M, Godfrey MK, Katrina BM, Heejung B, Harusha S, Samuel EK, John MC, Warren DJ, Daniel WF. Urogenital schistosomiasis in women of reproductive age in Tanzania's lake victoria region. Amer. J. of Tropic. Med. and Hygiene. 2011;84(3):364369.

29. Clements AC, Lwambo NJ, Blair L, Nyandindi U, Kaatano G, Kinung'hi S, Webster JP, Fenwick A, Brooker S. Bayesian spatial analysis and disease mapping: Tools to enhance planning and implementation of a schistosomiasis control programme in Tanzania. Tropic. Med. and Intern. Health. 2006; 11:490-503.
30. Swai B, Poggensee G, Mtweve S, Krantz I. Female genital schistosomiasis as an evidence of a neglected cause for reproductive ill-health: A retrospective histopathological study. BMC Infectious Disease. 2006;6:134.

31. Norseth HM, Ndhlovu PD, Kleppa E, Randrianasolo BS, Peter M, Jourdan PM, Roald B, Holmen SD, Gundersen SG, Bagratee J, Onsrud M, Kjetland EF. The Colposcopic Atlas of Schistosomiasis in the Lower Female Genital Tract Based on Studies in Malawi, Zimbabwe, Madagascar and South Africa; 2014.

32. IARC. Schistosoma haematobium International Agency for Research on Cancer (IARC) MONOGRAPHS - 100B; 2012.

(C) 2017 Gitau et al.; This is an Open Access article distributed under the terms of the Creative Commons Attribution License (http://creativecommons.org/licenses/by/4.0), which permits unrestricted use, distribution, and reproduction in any medium, provided the original work is properly cited. 